\title{
Integrated Topology Optimization of Structure/Vibration Control for Piezoelectric Cylindrical Shell Based on the Genetic Algorithm
}

\author{
Jianjun He and Xiangzi Chen \\ School of Automobile and Mechanical Engineering, Changsha University of Science and Technology, Changsha 410004, China \\ Correspondence should be addressed to Jianjun He; hezhengde8@163.com
}

Received 26 November 2014; Revised 29 March 2015; Accepted 10 April 2015

Academic Editor: Quoc Hung Nguyen

Copyright (C) $2015 \mathrm{~J}$. He and X. Chen. This is an open access article distributed under the Creative Commons Attribution License, which permits unrestricted use, distribution, and reproduction in any medium, provided the original work is properly cited.

\begin{abstract}
A hybrid optimization strategy for integrated topological optimization design of piezoelectric cylindrical flat shell structure is proposed. The method combines the genetic algorithm (GA) and linear-quadratic-regulator (LQR) theory to optimize the performance of coupling structure/control system. The GA is used to choose the optimal structure topology and number and placements of actuators and control parameters; meanwhile, the LQR is used to design control system to suppress vibration of optimal structure under sinusoidal excitation, which is based on the couple-mode space control. In addition, the mathematical morphology operators are used for repairs of disconnected structure topology. The results of numerical simulation and computations show that the proposed method is effective and feasible, with good performance for the optimal and coupling piezoelectric cylindrical shell structure/control system.
\end{abstract}

\section{Introduction}

The cylindrical shell structure is widely used as main parts of the large structure design in many fields, such as civil engineering and architecture, marine, and aerospace. For instance, the roofs of many stadiums and solid fuel tank of rocket are this type of structure due to their excellent mechanical properties, especially their high specific stiffness and strength [1]. However, the vibrations of those structures under complex and extreme dynamic loads are inevitable, as they are excited by strong wind and wave. So it is also necessary to design control system to suppress the amplitude of vibration for those structures in engineering practice $[2,3]$.

The optimization design can effectively improve various performances of structure; for example, it can make the optimal structure more lightweight and controllable. In particular, the topology optimization design has huge potential of saving material in structure design, which is verified by many applications of this technique in aerospace and automobile industry. For example, the application of topology optimization in the design of A380 leading edge ribs led to the forty-five percentage weight reduction compared to traditional design, and so forth. At present, smart or adaptive structures are widely used in industrial or engineering fields, so engineers can monitor and control the normal work of structures in real-time. However, because of the complexity of the adaptive structure, the structure and control system is coupled. For instance, the vibration controller's coefficients are strongly dependent on the topology of structure, the placements, and number of the actuators/sensors, which determine the effectiveness of structural vibration control. Therefore, researching the structure and control of integrated optimization strategies for smart structures is theoretically and practically significant. It can simultaneously realize the optimal design of structure and control system and simplify design links of huge and complex structure/control integrated system. So the integrated optimization design greatly improves the efficiency of traditional sequential optimization design (namely, the designer firstly optimizes the structure parameters and then optimizes the controller parameters).

Presently, for the active control of truss or plate and shell structures, piezoelectric patches and stacks or piezoelectric ceramic transducer (PZT) is used as one of the main actuators. For the optimization strategy, the GA is the main 
optimization algorithm. There have been some works published on intelligent piezoelectric structure integrated optimization. For instance, Li and Huang [4] combined the GA and the linear quadratic Gaussian (LQG), studied the integrated optimization of actuator placement and vibration control for piezoelectric adaptive trusses, and verified that the result of experiment is consistent with numerical results. Zhu et al. [5] investigated simultaneous optimization with respect to the structural topology, actuator locations, and control parameters of an actively controlled plate structure; its results showed that it can produce systems with clear structural topology and high control performance by the proposed approach. Kang and Tong [6] investigated the integrated optimization of structural topology and control voltage of piezoelectric laminated plates. Xu et al. [7] discussed some issues associated with integrated optimization of structural topology, the number and placement of actuators and control parameters for piezoelectric smart trusses; some optimal strategies based on genetic algorithms were adopted in their study. $\mathrm{Xu}$ et al. [8] studied the integrated optimization of structural topology and number and positions of the actuators and control parameters for piezoelectric smart plates, which is based on the optimal control effect in the independent mode control and singular value decomposition of the distributed matrix of total performance index for all physical control forces with the GA as optimization strategy. However, almost all the efforts described in the literatures involve the integrated optimization design of piezoelectric truss or plate, not including shell structure. In general, it is more complicated to construct the piezoelectric shell finite element for mathematical modeling and finite element analysis (FEA) for shell structure. Shell structure usually has more DOF and more complicated structural characteristic and mechanical behavior than truss and plate structure. Shell structure has more complicated geometric shape, which leads to its greater difficulties for modeling and analysis. If the shell structure is in vibration, its vibration suppression technique is usually invalid and more complicated than that of truss and plate structure. Thus, the main concern of this paper is to extend the integrated topology optimization design into shell structure.

In the present paper, the integrated topology optimization of structure/control for piezoelectric cylindrical flat shell is studied. Firstly, the piezoelectric coupling finite element (FE) model was built. Then the integrated optimization modeling, including the design variables, the objective function, and the constraint functions, was proposed, and the LQR was used for the design of control system in coupling modal space. In addition, in order to ensure the realization of integrated optimization, the GA and mathematical morphology treatment was applied to solution of the above problems. To verify the validity of the integrated optimization design of shell structure and promote engineering applications of this approach, the numerical simulations and computations were conducted. The results show that the integrated optimizations of piezoelectric cylindrical shell structure are successful and feasible.

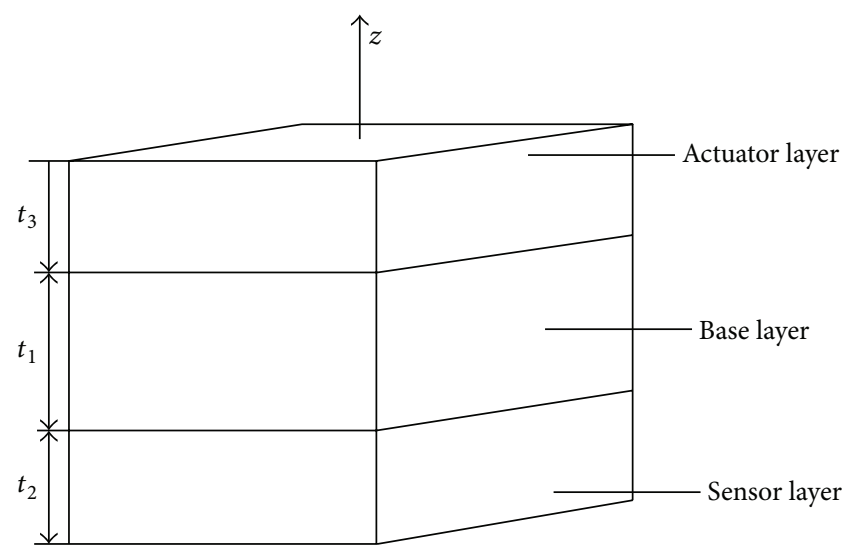

FIGURE 1: Four-node piezoelectric coupling rectangular flat shell element.

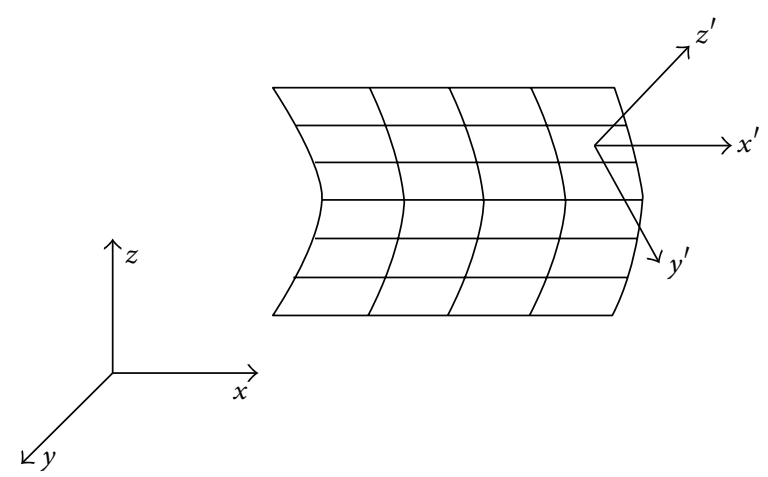

FIgURE 2: The coordinate system of cylindrical flat shell.

\section{The Piezoelectric Coupling Dynamic Model of Cylindrical Flat Shell Structure}

In general, it is more complicated to construct the piezoelectric shell finite element for mathematical modeling and finite element analysis (FEA) of shell structure. Shell structure usually has more DOF and more complicated structural characteristic and mechanical behavior than truss and plate structure. For simplification, we utilized the four-node rectangular flat shell element with 24 displacement degrees of freedom (DOF) and 2 electrical DOF to build the piezoelectric coupling dynamic model of cylindrical shell structure [3, 8]. The ideal element is shown in Figure 1. The piezoelectric flat shell element is composed of middle isotropic base layer with upper and lower surfaces of base layer that are completely covered by PZT patches, where $t_{1}$ and $t_{2 \sim 3}$ are the thickness of base layer and piezoelectric patches, respectively. The approximate cylindrical flat shell structure is shown in Figure 2.

In finite element derivation, the node displacement vector of flat shell structural element is defined as

$$
\mathbf{d}_{e i}^{\prime}=\left(\begin{array}{lllllll}
u_{i}^{\prime} & v_{i}^{\prime} & w_{i}^{\prime} & \theta_{x i}^{\prime} & \theta_{y i}^{\prime} & \theta_{z i}^{\prime}
\end{array}\right)^{\mathrm{T}} \quad i=1,2,3,4,
$$

where $i$ is denoted by the four nodes of the flat shell structural element. 
Assume that the element stiffness matrix corresponding to the plane stress state is expressed as

$$
\mathbf{k}_{e}^{m}=\left[\begin{array}{llll}
\mathbf{k}_{11}^{m} & \mathbf{k}_{12}^{m} & \mathbf{k}_{13}^{m} & \mathbf{k}_{14}^{m} \\
\mathbf{k}_{21}^{m} & \mathbf{k}_{22}^{m} & \mathbf{k}_{23}^{m} & \mathbf{k}_{24}^{m} \\
\mathbf{k}_{31}^{m} & \mathbf{k}_{32}^{m} & \mathbf{k}_{33}^{m} & \mathbf{k}_{34}^{m} \\
\mathbf{k}_{41}^{m} & \mathbf{k}_{42}^{m} & \mathbf{k}_{43}^{m} & \mathbf{k}_{44}^{m}
\end{array}\right] \text { node } \text { node 3 }
$$

where superscript " $m$ " is corresponding to the stiffness matrix of plane stress; $\mathbf{k}_{i j}^{m}$ is denoted by $2 \times 2$ submatrix.

Similarly, assume that the element stiffness matrix corresponding to the bending stress state is expressed as

$$
\mathbf{k}_{e}^{b}=\left[\begin{array}{cccc}
\mathbf{k}_{11}^{b} & \mathbf{k}_{12}^{b} & \mathbf{k}_{13}^{b} & \mathbf{k}_{14}^{b} \\
\mathbf{k}_{21}^{b} & \mathbf{k}_{22}^{b} & \mathbf{k}_{23}^{b} & \mathbf{k}_{24}^{b} \\
\mathbf{k}_{31}^{b} & \mathbf{k}_{32}^{b} & \mathbf{k}_{33}^{b} & \mathbf{k}_{34}^{b} \\
\mathbf{k}_{41}^{b} & \mathbf{k}_{42}^{b} & \mathbf{k}_{43}^{b} & \mathbf{k}_{44}^{b}
\end{array}\right] \text { node 1 }{ }_{\text {node } 2}^{\text {node 4 }}
$$

where superscript " $b$ " is corresponding to the stiffness matrix of bending stress; $\mathbf{k}_{i j}^{b}$ is denoted by $3 \times 3$ submatrix.

By combining with (2) and (3), the element stiffness matrix of flat shell structural element in local coordinate system is obtained as

$$
\begin{aligned}
& \mathbf{k}_{e}^{\prime} \\
& =\left[\begin{array}{ccccccccccccc}
\mathbf{k}_{11}^{m} & 0 & 0 & \mathbf{k}_{12}^{m} & 0 & 0 & \mathbf{k}_{13}^{m} & 0 & 0 & \mathbf{k}_{14}^{m} & 0 & 0 \\
0 & \mathbf{k}_{11}^{b} & 0 & 0 & \mathbf{k}_{12}^{b} & 0 & 0 & \mathbf{k}_{13}^{b} & 0 & 0 & \mathbf{k}_{14}^{b} & 0 \\
0 & 0 & 0 & 0 & 0 & 0 & 0 & 0 & 0 & 0 & 0 & 0 \\
\mathbf{k}_{21}^{m} & 0 & 0 & \mathbf{k}_{22}^{m} & 0 & 0 & \mathbf{k}_{23}^{m} & 0 & 0 & \mathbf{k}_{24}^{m} & 0 & 0 \\
0 & \mathbf{k}_{21}^{b} & 0 & 0 & \mathbf{k}_{22}^{b} & 0 & 0 & \mathbf{k}_{23}^{b} & 0 & 0 & \mathbf{k}_{24}^{b} & 0 \\
0 & 0 & 0 & 0 & 0 & 0 & 0 & 0 & 0 & 0 & 0 & 0 \\
\mathbf{k}_{31}^{m} & 0 & 0 & \mathbf{k}_{32}^{m} & 0 & 0 & \mathbf{k}_{33}^{m} & 0 & 0 & \mathbf{k}_{34}^{m} & 0 & 0 \\
0 & \mathbf{k}_{31}^{b} & 0 & 0 & \mathbf{k}_{32}^{b} & 0 & 0 & \mathbf{k}_{33}^{b} & 0 & 0 & \mathbf{k}_{34}^{b} & 0 \\
0 & 0 & 0 & 0 & 0 & 0 & 0 & 0 & 0 & 0 & 0 & 0 \\
\mathbf{k}_{41}^{m} & 0 & 0 & \mathbf{k}_{42}^{m} & 0 & 0 & \mathbf{k}_{43}^{m} & 0 & 0 & \mathbf{k}_{44}^{m} & 0 & 0 \\
0 & \mathbf{k}_{41}^{b} & 0 & 0 & \mathbf{k}_{42}^{b} & 0 & 0 & \mathbf{k}_{43}^{b} & 0 & 0 & \mathbf{k}_{44}^{b} & 0 \\
0 & 0 & 0 & 0 & 0 & 0 & 0 & 0 & 0 & 0 & 0 & 0
\end{array}\right],
\end{aligned}
$$

where $\mathbf{k}_{e}^{\prime}$ is derived in local coordinate system, which is a $24 \times$ 24 matrix.

By eliminating the singularity of the whole stiffness matrix of shell structure, we should transform all the local element stiffness matrices into global coordinate system by coordinate transformation.
The transformation matrix is defined as follows:

$$
\begin{aligned}
& \mathbf{T}=\left[\begin{array}{llllllll}
\varphi & 0 & 0 & 0 & 0 & 0 & 0 & 0 \\
0 & \varphi & 0 & 0 & 0 & 0 & 0 & 0 \\
0 & 0 & \varphi & 0 & 0 & 0 & 0 & 0 \\
0 & 0 & 0 & \varphi & 0 & 0 & 0 & 0 \\
0 & 0 & 0 & 0 & \varphi & 0 & 0 & 0 \\
0 & 0 & 0 & 0 & 0 & \varphi & 0 & 0 \\
0 & 0 & 0 & 0 & 0 & 0 & \varphi & 0 \\
0 & 0 & 0 & 0 & 0 & 0 & 0 & \varphi
\end{array}\right], \\
& \boldsymbol{\varphi}=\left[\begin{array}{lll}
\cos \left(x^{\prime}, x\right) & \cos \left(x^{\prime}, y\right) & \cos \left(x^{\prime}, z\right) \\
\cos \left(y^{\prime}, x\right) & \cos \left(y^{\prime}, y\right) & \cos \left(y^{\prime}, z\right) \\
\cos \left(z^{\prime}, x\right) & \cos \left(z^{\prime}, y\right) & \cos \left(z^{\prime}, z\right)
\end{array}\right],
\end{aligned}
$$

where $\mathbf{f}$ is denoted by direction cosine matrix of local coordinate system versus global coordinate system.

After coordinate transformation by (6), the local element stiffness matrix is transformed into global element stiffness matrix:

$$
\mathbf{k}_{e}=\mathbf{T}^{\mathrm{T}} \mathbf{k}_{e}^{\prime} \mathbf{T}^{\mathrm{T}}
$$

Finally, we can get the whole stiffness matrix in global coordinate system through elements assembly process.

By using the Hamilton principle, we can obtain the formula as follows:

$$
\delta \int_{t_{1}}^{t_{2}}\left(T^{e}-U^{e}+W^{e}\right) d t=0,
$$

where $T^{e}, U^{e}$, and $W^{e}$ are the kinetic energy, potential energy, and external work of the piezoelectric coupling flat shell element shown in Figure 1, respectively.

Substituting the kinetic energy, potential energy, and external work of the piezoelectric coupling flat shell element into (7), and calculating the variation of (7), the piezoelectric coupling equation is obtained as follows:

$$
\begin{aligned}
& \mathbf{M}^{e}\left\{\ddot{\mathbf{q}}^{e}\right\}+\left(\mathbf{K}_{p}^{e}+\mathbf{K}_{m}^{e}\right)\left\{\mathbf{q}^{e}\right\}-\mathbf{K}_{m a}^{e} V_{a z}^{e}+\mathbf{K}_{m s}^{e} V_{s z}^{e} \\
& \quad=\left\{F_{f}^{e}\right\}, \\
& \mathbf{K}_{a m}^{e}\left\{\mathbf{q}^{e}\right\}+\mathbf{K}_{a a}^{e} V_{a z}^{e}=-\iint_{A^{e}} \bar{\sigma}_{a}^{e} d x d y, \\
& \mathbf{K}_{s m}^{e}\left\{\mathbf{q}^{e}\right\}+\mathbf{K}_{s s}^{e} V_{s z}^{e}=0, \\
& \mathbf{M}^{e}=\sum_{h=m, s, a} \int_{V_{h}^{e}} \rho_{h}[\mathbf{N}]^{\mathrm{T}}[\mathbf{N}] d V, \\
& \mathbf{K}_{m}^{e}=\int_{V_{m}^{e}} z^{2}\left[\mathbf{B}_{u}\right]^{\mathrm{T}}\left[\mathbf{D}_{m}\right]\left[\mathbf{B}_{u}\right] d V,
\end{aligned}
$$




$$
\begin{aligned}
& \mathbf{K}_{p}^{e}=\int_{V_{s}^{e}+V_{a}^{e}} z^{2}\left[\mathbf{B}_{u}\right]^{\mathrm{T}}\left[\mathbf{S}^{E}\right]\left[\mathbf{B}_{u}\right] d V, \\
& \mathbf{K}_{m a}^{e}=\left(\mathbf{K}_{a m}^{e}\right)^{\mathrm{T}}=\int_{V_{a}^{e}} \frac{z\left[\mathbf{B}_{u}\right]^{\mathrm{T}}[\mathbf{e}]^{\mathrm{T}}}{t_{2}} d V, \\
& \mathbf{K}_{m s}^{e}=\left(\mathbf{K}_{s m}^{e}\right)^{\mathrm{T}}=\int_{V_{s}^{e}} \frac{z\left[\mathbf{B}_{u}\right]^{\mathrm{T}}[\mathbf{e}]^{\mathrm{T}}}{t_{2}} d V, \\
& \mathbf{K}_{a a}^{e}=\mathbf{K}_{s s}^{e}=\int_{V_{a}^{e}} \frac{\varepsilon_{33}^{E}}{t_{2}^{2}} d V, \\
& \left\{\mathbf{F}_{f}^{e}\right\}=\iint_{A_{e}} \mathbf{N}^{\mathrm{T}} \mathbf{f} d x d y,
\end{aligned}
$$

where $\mathbf{q}^{e}$ is denoted by node displacement vector of the piezoelectric coupling flat shell element, and the meanings of the other symbols are similar to the utilizations of these in the literature $[3,9]$.

By reducing the $V_{s z}^{e}$ and $V_{a z}^{e}$ terms in (8)-(10), the piezoelectric coupling finite element equation is obtained as follows:

$$
\mathbf{M}^{e}\left\{\ddot{\mathbf{q}}^{e}\right\}+\mathbf{K}^{e}\left\{\mathbf{q}^{e}\right\}=\left\{\mathbf{F}_{f}^{e}\right\}-\left\{\mathbf{F}_{\text {control }}^{e}\right\}
$$

where

$$
\begin{aligned}
\mathbf{K}^{e}= & \mathbf{K}_{m}^{e}+\mathbf{K}_{p}^{e}+\left(\mathbf{K}_{m a}^{e}\right)^{\mathrm{T}}\left(\mathbf{K}_{a a}^{e}\right)^{-1} \mathbf{K}_{a m} \\
& +\left(\mathbf{K}_{m s}^{e}\right)^{\mathrm{T}}\left(\mathbf{K}_{s s}^{e}\right)^{-1} \mathbf{K}_{s m} .
\end{aligned}
$$

The equivalent control force of input voltage in actuator layer is calculated as

$$
\left\{\mathbf{F}_{\text {control }}^{e}\right\}=\mathbf{K}_{m a}^{e}\left(\mathbf{K}_{a a}^{e}\right)^{-1} \iint_{A^{e}} \frac{\varsigma_{0}}{t_{2}} V_{a z}^{e} d x d y .
$$

Similarly, we can finally establish the piezoelectric coupling dynamic FE equation for the global coupled piezoelectric structure/control system through elements stiffness matrix assembly process as follows:

$$
\mathbf{M} \ddot{\mathbf{q}}(t)+\mathbf{K q}(t)=\mathbf{F}_{\text {load }}(t)+\mathbf{F}_{\text {control }}(t),
$$

where $\mathbf{M}$ and $\mathbf{K}$ are the coupled system mass matrix and system stiffness matrix, respectively; $\mathbf{q}$ and $\ddot{\mathbf{q}}$ are the structure nodal displacement vector and acceleration vector, respectively; $\mathbf{F}_{\text {load }}(t)$ and $\mathbf{F}_{\text {control }}(t)$ are the load vector and control force vector, respectively. $\mathbf{F}_{\text {control }}(t)$ can be expressed as $\mathbf{B u}(t)$, where $\mathbf{B}$ and $\mathbf{u}(t)$ are the actuator distributing matrix and the control force vector, respectively. In (21), we did not consider the damping term in order to emphasize the vibration suppression by active control. This section is used for modeling and finite element analysis of piezoelectric coupling flat shell structure and is followed by the LQR control design in the integrated optimization procedure.

\section{The Mathematical Modeling for Integrated Optimization Design of Piezoelectric Cylindrical Shell Structure}

The standard form of the integrated optimization problem for piezoelectric smart cylindrical flat shell structure can be described as

$$
\begin{array}{cl}
\text { find } & d_{i}^{s}, d_{k}^{c} \quad(i=1,2, \ldots, N ; k=1,2, \ldots, M) \\
\min & S=\sum_{i=1}^{N} 4 \rho_{i} a_{i} b_{i} t_{i} \\
\text { s.t } \quad & g\left(d_{i}^{s}, d_{k}^{c}\right)_{j} \leq 0 \quad(j=1,2, \ldots, p)
\end{array}
$$

where $d_{i}^{s}$ and $d_{k}^{c}$ are the structural design variable and control design variable, respectively; to minimize the structure/control system mass $S$ is the objective function; $N, \rho_{i}$, $a_{i}, b_{i}$, and $t_{i}$ are the number of elements (including ordinary elements and piezoelectric coupling elements), the density, half-length of long side and short side for rectangular flat shell element, and thickness of structure element or piezoelectric element, respectively; $m, n$ are the number of structural design variable and control design variable, respectively; $g\left(d_{i}^{s}, d_{k}^{c}\right)_{j}$ is coupled constraint functions, which means it is simultaneously in relation to the structural design variable and control design variable.

3.1. Design Variables. The topology design variable $d_{i}^{c}$ indicates the presence or absence of structure material: that is,

$$
d_{i}^{s} \in\{0,1\}
$$

if $d_{i}^{s}=1$ indicates that the corresponding element exists, or else it is removed in optimization process. On the contrary, $d_{i}^{s}=0$ indicates that it is a void element without material.

The elements of $d_{k}^{c}$ may be the control design parameters and the number and positions of actuators (PZT patches):

$$
d_{k}^{c}= \begin{cases}n_{a}, & n_{a} \in[1, S] \\ p_{n_{a}}, & p_{n_{a}} \in[1, N] \\ R_{n_{a}}, & R_{n_{a}} \in\left[R^{l}, R^{u}\right],\end{cases}
$$

where $n_{a}, p_{n_{a}}$, and $R_{n_{a}}$ are the number of actuators, the positions of actuators, and weight factor of control weight matrix, respectively. $S, N, R^{l}$, and $R^{u}$ indicate the allowable maximal number of actuators, the allowable maximal element position of actuators, and the lower bound and upper bound of weight factor, respectively.

3.2. Constraint Function. Here, the constraint functions contain the first natural frequency constraint function, voltage constraint function, and weight factor constraint function.

3.2.1. Natural Frequency Constraint Function. In engineering practice, structure usually suffers from dynamic load, which may cause resonance or coupled effect between the structure and external excitation. These cases should be avoided 
because of their huge destruction to structure. In general, we impose natural frequency constraint to the optimization mathematical modeling, especially for the first natural frequency. So the natural frequency constraint is expressed as

$$
f_{1}^{I}-f_{1}^{R} \leq 0
$$

where $f_{1}^{I}$ and $f_{1}^{R}$ are the first anticipant minimal natural frequency of optimization structure and the first actual natural frequency of optimal structure, respectively.

3.2.2. Voltage Constraint Function. Presently, piezoelectric patches or PZT are used as one of the main actuators in active vibration control of structure. However, there is a breakover voltage for the piezoelectric patches and the applied voltage by the voltage amplifier is limited; the active control system cannot provide the unlimited and necessary control force beyond the break-over voltage. Then the constraint on the active control force can be transformed into the corresponding voltage constraint: that is,

$$
V_{k}^{a}-\bar{V}_{k}^{a} \leq 0
$$

where $V_{k}^{a}$ and $\bar{V}_{k}^{a}$ are the actual applied voltage and its corresponding allowed upper limit, respectively.

3.2.3. Weight Factor Constraint Function. In LQR control, the selections of control weight matrixes have great impact on the velocity of response suppression and size of control force. So we choose weight factor as control design parameter and make it confined to a specified range: that is,

$$
R_{k}^{l} \leq R_{k} \leq R_{k}^{u}
$$

where $R_{k}^{l}$ and $R_{k}^{u}$ are the corresponding lower bound and upper bound of control weight factor, respectively.

\section{LQR Control Modeling and Design}

In modern control theory, LQR is one of the mature and effective optimal controller's design methods, which is based on state space expression and is applied in many literatures, such as in the literature [8]. In general, the vibration suppression of flat shell structure is more complicated than truss and plate structure. For simplifications, we still utilize the LQR for control modeling and design here. In the following, the LQR approach for design of control system is given out.

By considering the first several waiting control modes and combining with the initial conditions, after modal coordinate transformation, (21) can be rewritten as in the modal space:

$$
\begin{aligned}
& \left\{\begin{array}{c}
\ddot{\mathbf{y}}_{c}(t) \\
\ddot{\mathbf{y}}_{r}(t)
\end{array}\right\}+\left[\begin{array}{cc}
\boldsymbol{\Lambda}_{c} & 0 \\
0 & \boldsymbol{\Lambda}_{r}
\end{array}\right]\left\{\begin{array}{c}
\mathbf{y}_{c}(t) \\
\mathbf{y}_{r}(t)
\end{array}\right\}=\left\{\begin{array}{c}
\phi_{c}^{\mathrm{T}} \\
\phi_{r}^{\mathrm{T}}
\end{array}\right\} \mathbf{B u}(t), \\
& \phi_{c}=\left[\begin{array}{llll}
\phi_{1} & \phi_{2} & \cdots & \phi_{n_{m}}
\end{array}\right] \text {, } \\
& \varphi_{i}^{\mathrm{T}} M \varphi_{j}=\delta_{i j} \text {, } \\
& \Lambda_{c}=\operatorname{diag}\left(\omega_{1}^{2}, \omega_{2}^{2}, \ldots, \omega_{n_{m}}^{2}\right) \text {, }
\end{aligned}
$$

where $\mathbf{y}_{c}(t)$ and $\mathbf{y}_{r}(t)$ are denoted by the modal displacement vector for waiting control and the rest modal displacement vector, respectively; $\boldsymbol{\Lambda}_{c}$ is expressed as the eigenvalues diagonal matrix for waiting control modes.

Equation (28) can be expressed as in state space with matrix form:

$$
\begin{aligned}
\dot{\mathbf{Z}}(t) & =\mathbf{A Z}(t)+\mathbf{B}^{\prime} \mathbf{u}(t), \\
\mathbf{Z}(t) & =\left[\begin{array}{l}
\mathbf{y}_{c}(t) \\
\dot{\mathbf{y}}_{c}(t)
\end{array}\right], \\
\mathbf{A} & =\left[\begin{array}{cc}
0_{n_{m} \times n_{m}} & \mathbf{I}_{n_{m} \times n_{m}} \\
-\boldsymbol{\Lambda}_{\mathbf{c}} & 0_{n_{m} \times n_{m}}
\end{array}\right], \\
\mathbf{B}^{\prime} & =\left[\begin{array}{c}
\mathbf{0}_{n_{m} \times n_{a}} \\
\boldsymbol{\varphi}_{c}^{\mathrm{T}} \mathbf{B}
\end{array}\right] .
\end{aligned}
$$

To ensure that the waiting control system is stable and controllable, the allocation number of actuators $n_{m}$ in coupled structure/control system should be met with the condition as

$$
\operatorname{rank}\left[\begin{array}{lllll}
\mathbf{B}^{\prime} & \mathbf{A B}^{\prime} & \mathbf{A}^{2} \mathbf{B}^{\prime} & \cdots & \mathbf{A}^{2 n_{m}-1} \mathbf{B}^{\prime}
\end{array}\right]=2 n_{m},
$$

where rank[] is denoted as the rank computation of matrix.

For obtaining the quadratic form optimal control design $\mathbf{u}(t)$ is to minimize the objective function $J$ :

$$
\begin{aligned}
J & =\frac{1}{2} \int_{0}^{\infty}\left(\mathbf{Z}^{\mathrm{T}}(t) \mathbf{Q Z}(t)+\mathbf{u}^{\mathrm{T}}(t) \mathbf{R} \mathbf{u}(t)\right) d t \\
& \longrightarrow \min , \\
\mathbf{Q} & =\left[\begin{array}{cc}
\boldsymbol{\Lambda}_{c} & 0_{n_{m} \times n_{m}} \\
0_{n_{m} \times n_{m}} & \mathbf{I}_{n_{m} \times n_{m}}
\end{array}\right], \\
\mathbf{R} & =\left[\begin{array}{ccc}
r_{1} & 0 \\
& \ddots & \\
0 & & r_{n_{a}}
\end{array}\right],
\end{aligned}
$$

where $\mathbf{R}$ is expressed as the control design parameters matrix.

To minimize (33), we can obtain $\mathbf{u}(t)$ as

$$
\mathbf{u}(t)=-\mathbf{R}^{-1}\left(\mathbf{B}^{\prime}\right)^{\mathrm{T}} \mathbf{P Z}(t),
$$

where $\mathbf{P}$ is a $2 n_{m} \times 2 n_{m}$ semidefinite and symmetric matrix, which satisfied the Riccati equation as follows:

$$
\mathbf{P A}+\mathbf{A}^{\mathrm{T}} \mathbf{P}+\mathbf{Q}-\mathbf{P B}^{\prime} \mathbf{R}^{-1}\left(\mathbf{B}^{\prime}\right)^{\mathrm{T}} \mathbf{P}=0
$$

By substituting (35) into (30), we can obtain the state equation of closed-loop system as

$$
\begin{aligned}
\dot{\mathbf{Z}}(t) & =\overline{\mathbf{A}} \mathbf{Z}(t), \\
\overline{\mathbf{A}} & =\mathbf{A}-\mathbf{B}^{\prime} \mathbf{R}^{-1}\left(\mathbf{B}^{\prime}\right)^{\mathrm{T}} \mathbf{P} .
\end{aligned}
$$

Thus, this section is used for calculating the optimal control force vector $\mathbf{u}(t)$ by (35) and determining the optimal control design parameters for (33). 


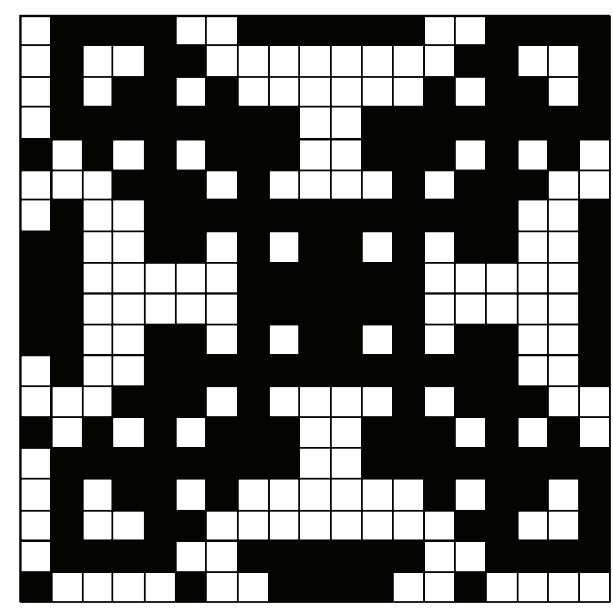

(a) Original topology

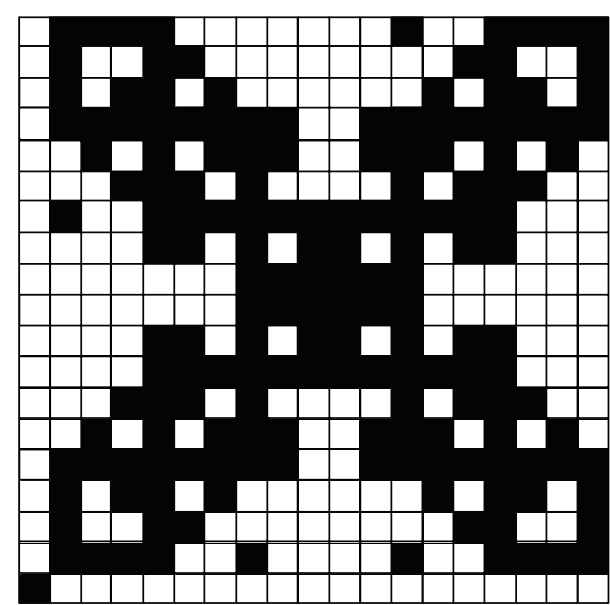

(b) Final topology

FIgURE 3: Effect for deletions of some low-stress elements.

\section{Integrated Optimization Algorithm and Strategy}

5.1. Optimization Algorithm. The problem in (22) has continuous and discrete design variables, which lead to its solution being more difficult and complex than ordinary optimization problem with single type's design variable. For the sake of coupling between the structure and control system and complexion of constraint functions, it is very difficult to find the optimum solution for combined optimization design problem with structural topology and control parameter. Generally, there exist several equal or not equal suboptimal solutions. For the saving of computational cost and simplifying of optimization design, we can find the suboptimal solution instead. Because of wide adaptability of the GA to the different types of optimization problems, then it may be an effective method for the solution of the above problem. So the GA is applied for the integrated optimization design in this paper. The binary is selected for coding of chromosome with structural design variable and control parameter design variable [8]. Meanwhile, the individual fitness for minimizing the objective function is expressed as

$$
\operatorname{eval}\left(d_{i}^{s}, d_{k}^{c}\right)=\frac{1}{S} \times\left(1+\text { penal } \times \frac{\sum_{j=1}^{p} D_{j}}{p}\right),
$$

where $p$ is the number of constraints functions and penal is the penalty factor. For natural frequency constraint function, $D_{1}=\left(f_{1}^{R}-f_{1}^{I}\right) / f_{1}^{I}$, the other $D_{j}$ are obtained by similar method as $D_{1}$.

5.2. Deletions of Some Low-Stress Elements. There generally exist massive low-stress or inefficient material elements in topological optimizing structure at each iteration because of control requirements realization for integrated design of coupled structure/control system. Those elements make the maximal lightweight potential of structure be not achieved by topology optimization. Here we delete a part of low-stress

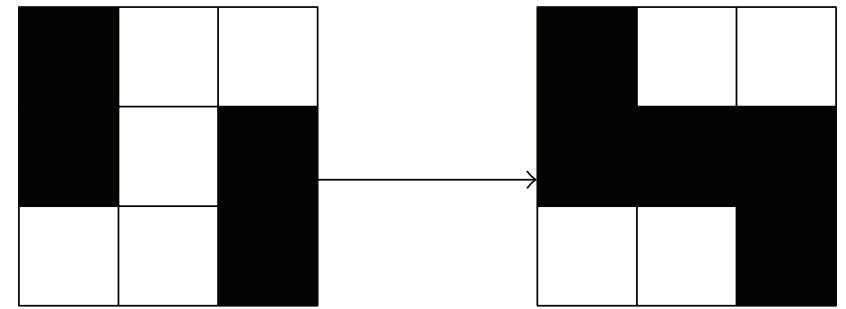

FIgURE 4: Morphology bridge operator.

elements at each iteration to make the topology structure be more clear and lightweight. The steps of the above strategy are summarized as follows: firstly, according to results of von Mises stress computation, we sort the whole elements of structure in ascending order; secondly, we delete certain percentages of low-stress elements. The deletion percentages are usually about $20 \%$. For example, let us see a comparison between the original topology and final topology treated by deletions of some low-stress elements, which is shown in Figure 3.

5.3. Repair Strategy for Disconnected Topology Based on Morphology Operator. The literature [10] points out that some disconnected topologies created by genetic algorithm commonly appear. The disconnected topology configuration is invalid and uncontrollable for integrated optimization design of structure/control system. So the repair strategy based on image mathematical morphology operators is applied to the disconnected topology in integrated optimization design in this paper. Firstly, topology of structure or topology variable matrix is considered as a gray image with solid and void areas. Then we use basic morphology operators "bridge" and "dilate" to filter the gray image that is obtained from each iteration. The detailed morphology operators and related formulations are expressed in the literature [11]. At first, let us simply understand the effects of two morphology operators, which are shown in Figures 4 and 5. 


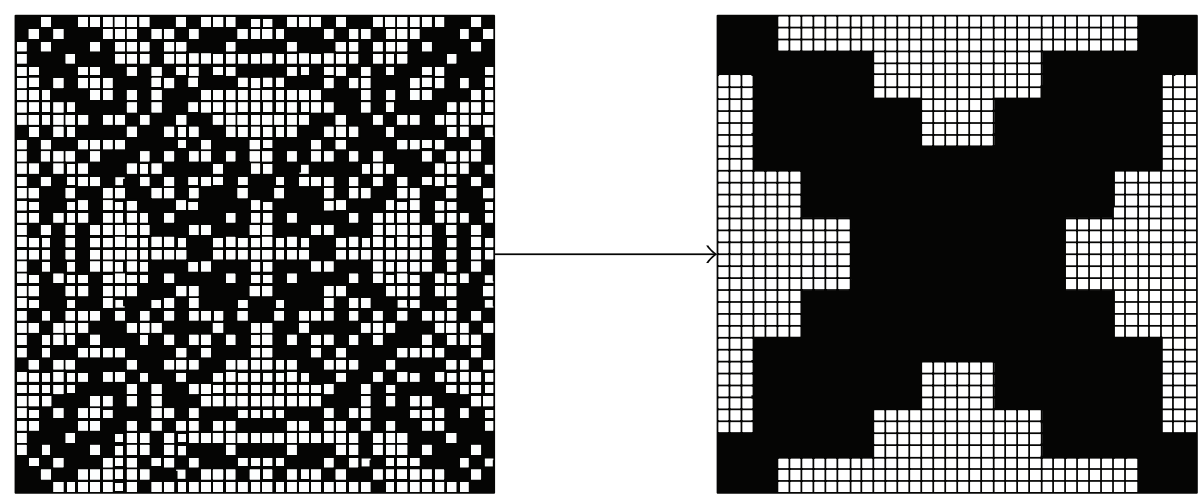

FIGURE 5: Morphology dilate operator for a piezoelectric smart plate with four simple supported corners.

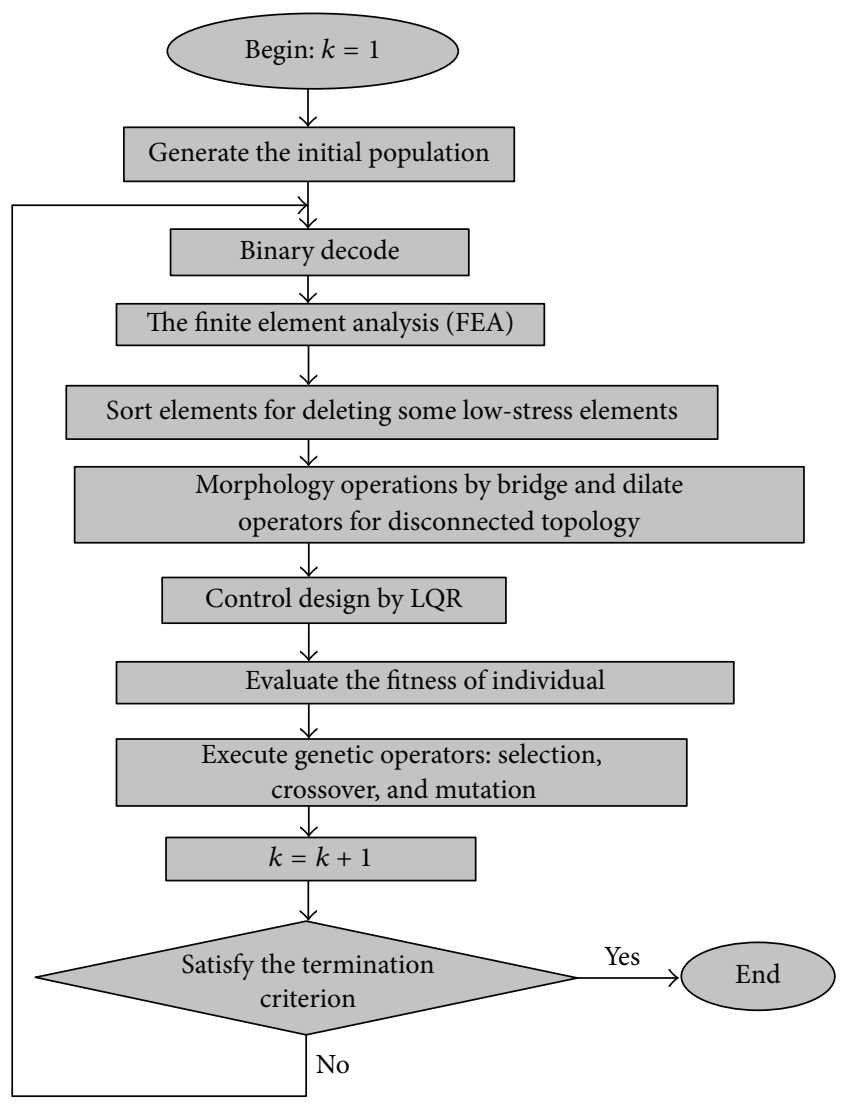

FIGURE 6: Flowchart for integrated optimization design of flat shell.

\section{The Integrated Optimization Design Steps of Structure/Control}

The detailed steps of integrated topology optimization design for piezoelectric cylindrical flat shell structure/control are shown in Figure 6.

The general genetic algorithm, LQR and FEA, was utilized for integrated topology optimization design for piezoelectric cylindrical flat shell structure/control in this paper, which appeared in many literatures [7-9]. Some steps in flowchart such as deletions of some low-stress elements and repair

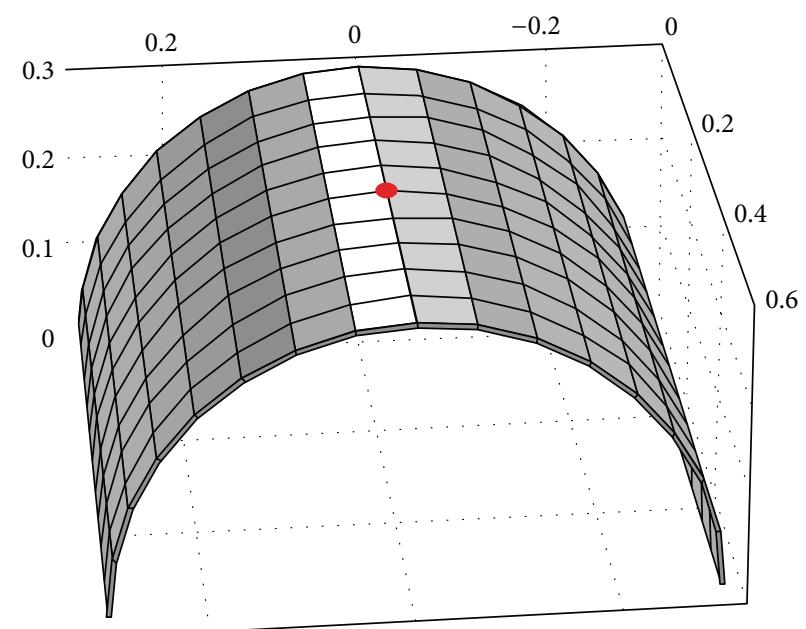

FIGURE 7: The original flat shell structure and the appointed node (labeled with red color).

strategy for disconnected topology based on morphology operator are described in Sections 5.2 and 5.3.

\section{Numerical Examples}

The simulation computations for two piezoelectric cylindrical flat shell structures are carried out by the proposed method. Here, the base material for two simulated structures is used by steel, and its material properties are as follows: Young's elastic modulus $E=2.1 \times 10^{11} \mathrm{~N} / \mathrm{m}^{2}$, Poisson's ratio $v=0.3$, and density $\rho=7800 \mathrm{~kg} / \mathrm{m}^{3}$. Meanwhile, the material properties of PZT patches are as follows: Young's elastic modulus $E_{p}=6.3 \times 10^{10} \mathrm{~N} / \mathrm{m}^{2}$, Poisson's ratio $v_{p}=0.3$, and density $\rho_{p}=7600 \mathrm{~kg} / \mathrm{m}^{3}$. Piezoelectric strain constants $d_{31}=d_{32}=-171 \times 10^{-12} \mathrm{C} / \mathrm{N}$, and dielectric constant $\varepsilon=15 \mathrm{nf} / \mathrm{m}$.

7.1. Semicylindrical Piezoelectric Shell Structure. The semicylindrical shell structure is shown in Figure 7. The structure is discretized to $10 \times 16$ elements, with the left and right edges being fixed, and the other edges are free. The sizes of 


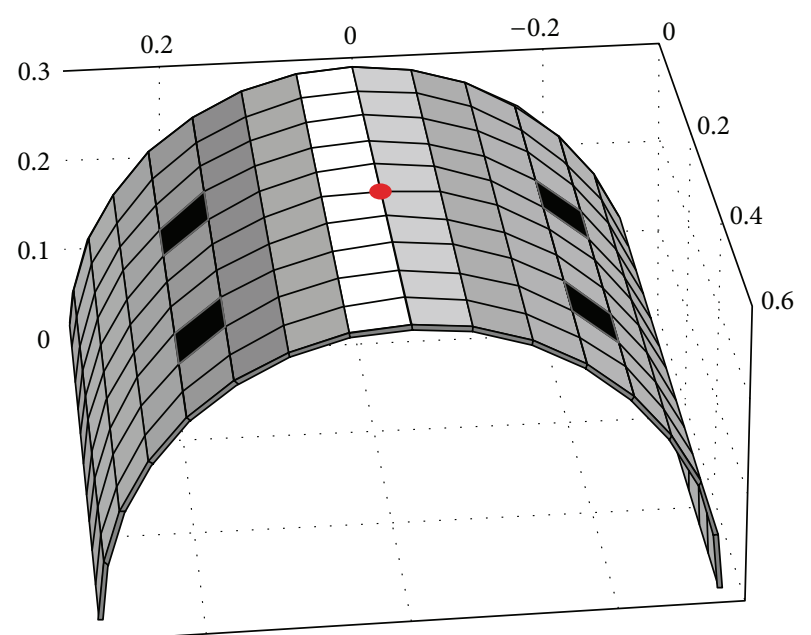

FIGURE 8: The optimal number and positions of PZT patches (labeled with black color).

TABLE 1: The results for integrated optimization design of semicylindrical shell.

\begin{tabular}{lccccc}
\hline$f_{1} / \mathrm{Hz}$ & $V_{1} / \mathrm{V}$ & $V_{2} / \mathrm{V}$ & $V_{3} / \mathrm{V}$ & $V_{4} / \mathrm{V}$ & $S(\mathrm{Kg})$ \\
\hline 19.00 & 39.208 & 39.208 & 62.244 & 62.244 & 7.3553 \\
\hline
\end{tabular}

structure are as follows: the length along generatrix direction is $L=0.6 \mathrm{~m}$, the ring radius is $R=0.3 \mathrm{~m}$, and the thickness is $t_{b}=0.004 \mathrm{~m}$. The thickness of PZT patches is $t_{p}=$ $0.001 \mathrm{~m}$. The static load condition is that the load of $1000 \mathrm{~N}$ is imposed in the negative $z$-direction of the appointed node, which is shown in Figure 7. The genetic algorithm parameters are as follows: population size, the crossover rate, and the mutation rate are $40,0.8$, and 0.02 , respectively. The allowable maximal iterations and the penalty factor are 200 and 1000, respectively.

The vibration of the cylindrical shell structure is caused by the sinusoidal excitation $F=1000 * \sin (2 * p i * 15 * t)$ that is imposed in the negative $z$-direction of the same node as the static load condition. The amplitude of applied voltages is not more than $100 \mathrm{~V}$; the allowable maximal number of piezoelectric elements or actuators is not more than 6 . We hope that we can use the several PZT actuators that are not more than 6 to control the six lower-order natural modes of the optimization structure. The frequency constraint is that the first natural frequency of the optimal structure is not less than $15 \mathrm{~Hz}$. The upper and lower bounds for control weight parameters are $1.0 \times 10^{-12}$ and $1.0 \times 10^{-13}$, respectively. The optimal number and positions of PZT patches are shown in Figure 8.

In Figure 8, the black blocks are piezoelectric coupling elements as expressed in Figure 1.

The results of integrated topology optimization design for semicylindrical shell structure/control are shown in Table 1.

The optimal structure topology is shown in Figure 9.

In Figure 9, the black and gray block areas are the solid elements and void elements, respectively. The optimal control

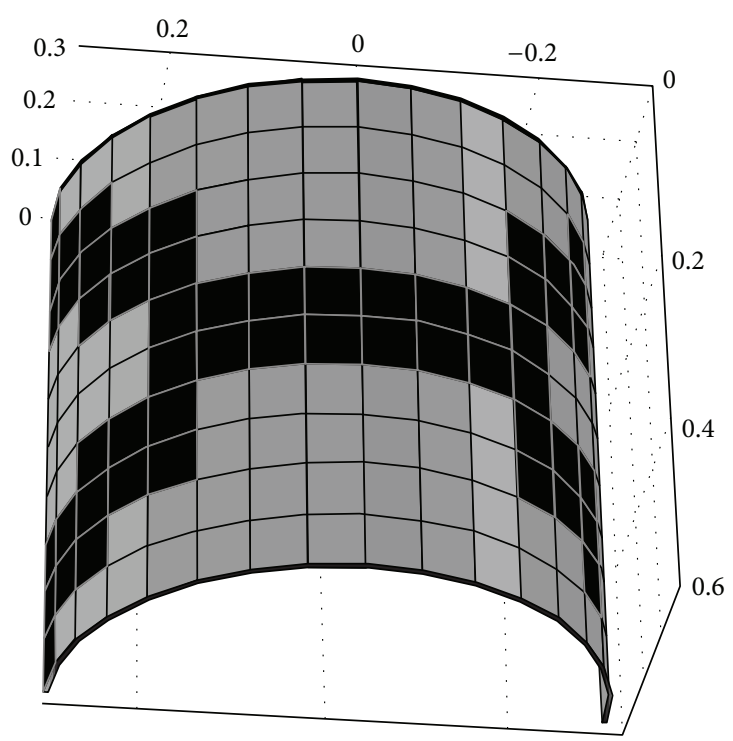

FIgURE 9: The optimal structure topology.

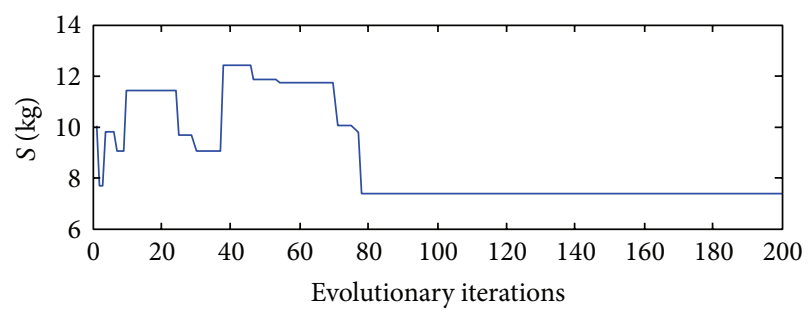

FIgURE 10: The iteration history of mass index.

weight parameters are $R_{a_{1,2}}=0.9789 \times 10^{-12}$ and $R_{a_{3,4}}=$ $0.4155 \times 10^{-12}$, respectively. The iteration history of mass index for the coupled structure/control is shown in Figure 10.

The vibration responses comparisons between under uncontrol condition and under integrated optimization control condition for this shell structure is shown in Figure 11.

7.2. Cantilever Piezoelectric Cylindrical Shell Structure. The cantilever cylindrical shell structure is shown in Figure 12. The structure is discretized to $10 \times 32$ elements, with the front ring edges being fixed, and the other edges are free. The sizes of structure are as follows: the length along generatrix direction is $L=0.8 \mathrm{~m}$, the ring radius is $R=0.4 \mathrm{~m}$, and the base thickness is $t_{b}=0.001 \mathrm{~m}$. The thickness of PZT patches is $t_{p}=0.0015 \mathrm{~m}$. The static load condition is that the load of $10000 \mathrm{~N}$ is imposed in the negative $z$ direction of the specified node, which is shown in Figure 11. The genetic algorithm parameters are as follows: population size, the crossover rate, and the mutation rate are $40,0.8$, and 0.02 , respectively. The allowable maximal iterations and the penalty factor are 100 and 10000 , respectively.

The vibration of the cantilever cylindrical shell structure is caused by the sinusoidal excitation $F=10000 * \sin (2 *$ $p i * 17.5 * t$ ) that is imposed in the negative $z$-direction of the same node as the static load condition. The amplitude 


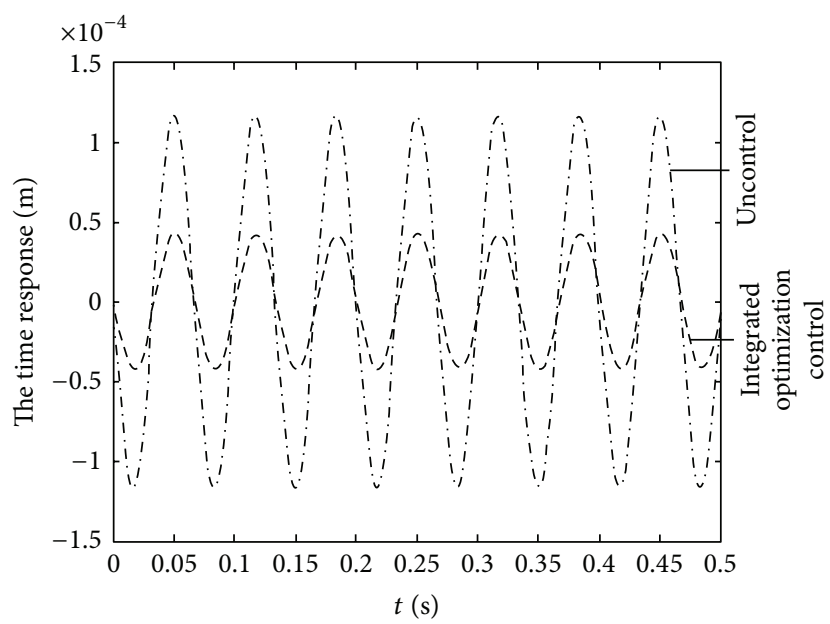

FIGURE 11: The time response of vibration structure at the excitation node along negative $z$-direction.

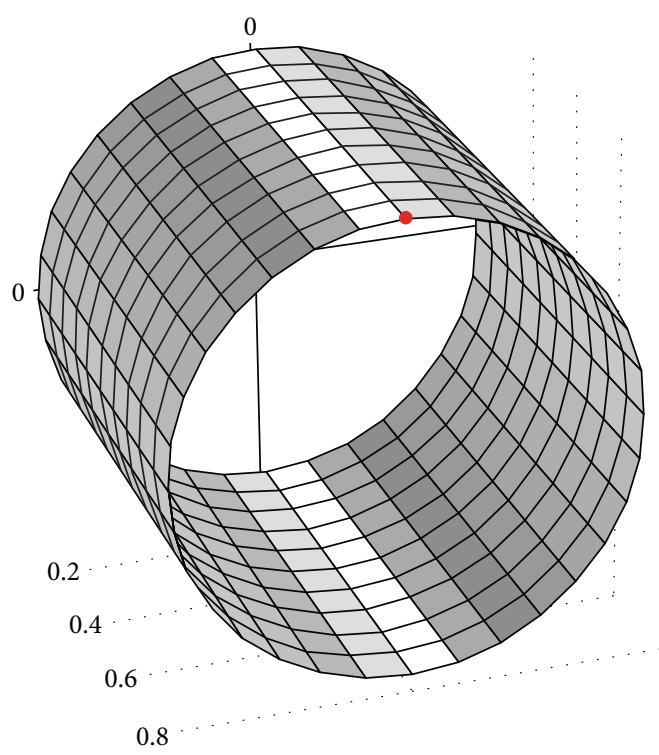

Figure 12: The original flat shell structure and the specified node (labeled with red color).

of applied voltages is not more than $100 \mathrm{~V}$; the allowable maximal number of PZT patches is not more than 6 . We hope that we can use the several PZT patches that are not more than 6 to control the six lower-order natural modes of the optimization structure. The frequency constraint is that the first natural frequency of the optimal structure is not less than $2 \mathrm{~Hz}$. The upper and lower bounds for control weight parameters are $1.0 \times 10^{-8}$ and $1.0 \times 10^{-9}$, respectively. The optimal number and positions of PZT patches are shown in Figure 13. The four piezoelectric flat shell elements are 55, 66, 96 , and 105 , respectively.

The results of integrated optimization design for cantilever cylindrical shell structure/control are shown in Table 2.

The optimal structure topology is shown in Figure 14.

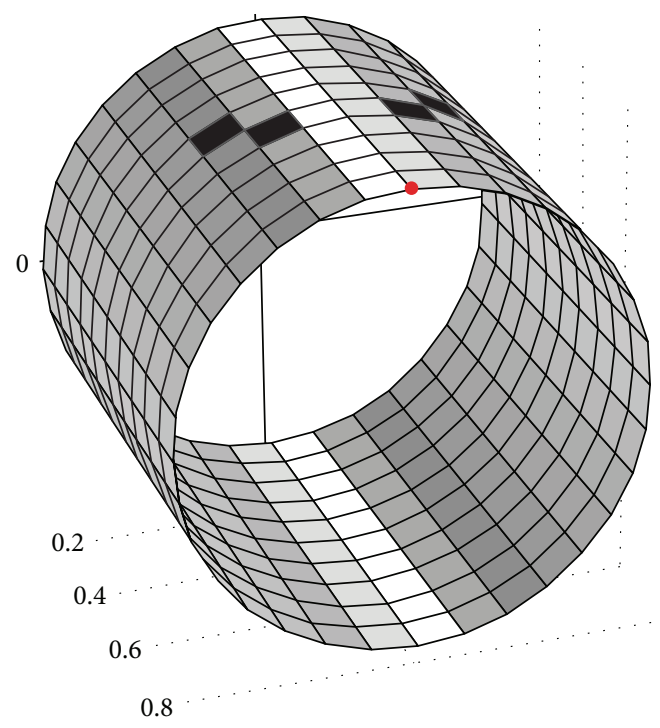

Figure 13: The optimal number and positions of PZT patches.

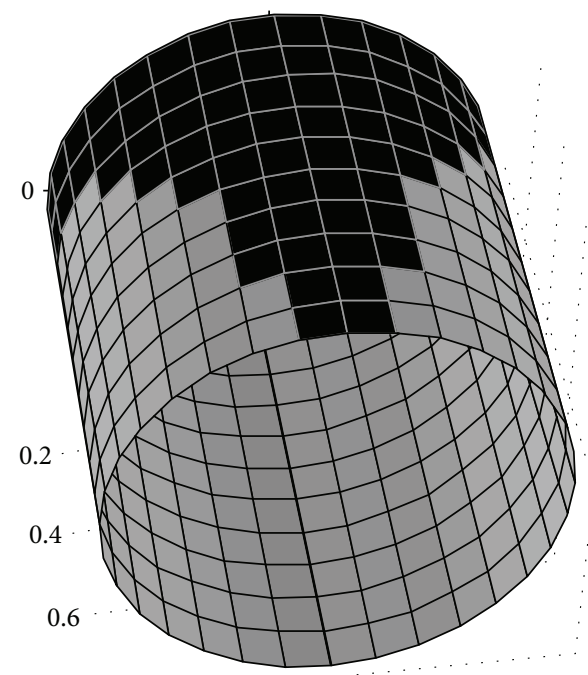

0.8

FIGURE 14: The optimal structure topology.

TABLE 2: The results for integrated optimization design of cantilever cylindrical shell.

\begin{tabular}{lccccc}
\hline$f_{1} / \mathrm{Hz}$ & $V_{1} / \mathrm{V}$ & $V_{2} / \mathrm{V}$ & $V_{3} / \mathrm{V}$ & $V_{4} / \mathrm{V}$ & $S(\mathrm{Kg})$ \\
\hline 2.886 & 8.842 & 8.842 & 24.806 & 24.806 & 7.8205 \\
\hline
\end{tabular}

The optimal control weight parameters are $R_{a_{1,2}}=$ $0.9561 \times 10^{-8}$ and $R_{a_{3,4}}=0.7574 \times 10^{-8}$, respectively. The iteration history of mass index for the coupling structure/control is shown in Figure 15.

The vibration responses comparisons between under uncontrol condition and under integrated optimization control condition for this shell structure is shown in Figure 16. 


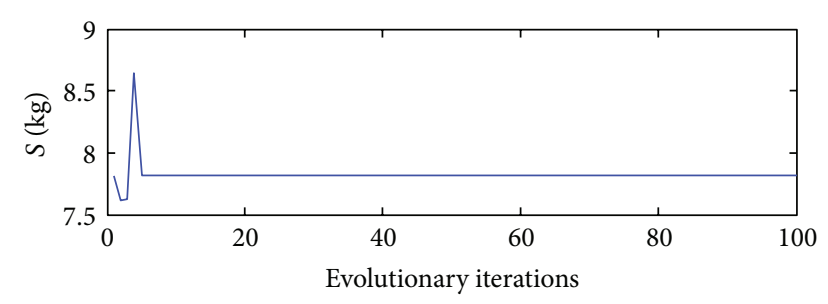

FIGURE 15: The iteration history of mass index.

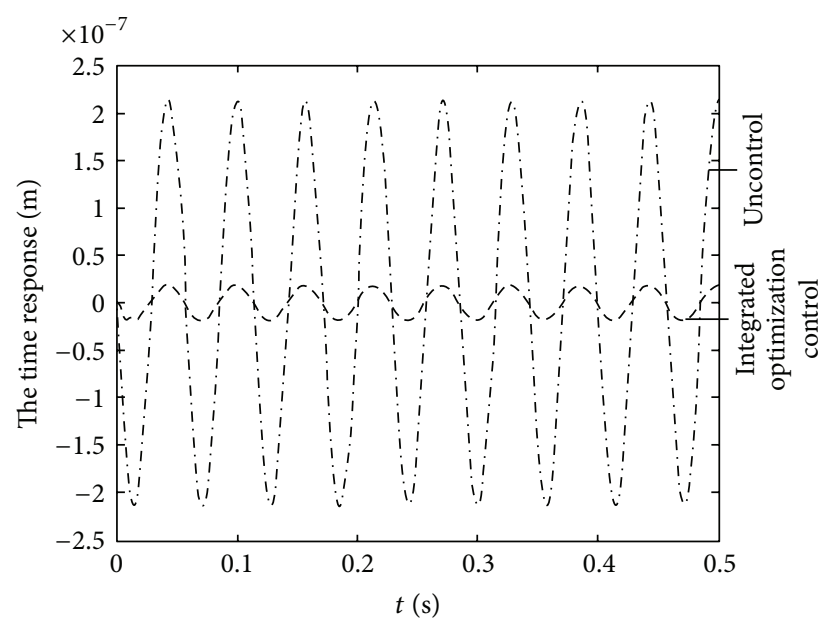

FIGURE 16: The time response comparisons at the excitation node along negative $z$-direction.

\section{Conclusions}

In order to enlarge the scope of application of the integrated optimization design of structure/control in engineering and practice, the integrated topology optimization design of piezoelectric cylindrical flat shell structure/control is studied in this paper. Firstly, the piezoelectric flat shell element is used for dynamic modeling of piezoelectric cylindrical shell structure. Secondly, based on the LQR control in coupling modal space, the integrated topology optimization design modeling with the GA is presented. Meanwhile, the strategies with the deletions of some low-stress elements and morphology operators are used for realization of integrated optimization design and smooth structure topology. Finally, numerical simulation computations are carried out for two cylindrical flat shell structures.

The results of numerical examples show that the proposed method can produce clear structure topology and high control performance. In two examples, the number and the positions of PZT patches are reasonable, and the amplitude of applied voltages is small, which are valuable to the realization and efficiency of integrated topology optimization design of piezoelectric cylindrical flat shell structure in engineering practice.

\section{Conflict of Interests}

The authors declare that there is no conflict of interests regarding the publication of this paper.

\section{Acknowledgments}

The authors would like to acknowledge the support by Natural Science Foundation of China under Grant 51305048 and Scientific Research Fund of Hunan Provincial Education Department under Grant 11C0045.

\section{References}

[1] P. Vidal, L. Gallimard, and O. Polit, "Shell finite element based on the Proper Generalized Decomposition for the modeling of cylindrical composite structures," Computers \& Structures, vol. 132, pp. 1-11, 2014.

[2] Y. Zhang, H. Niu, S. Xie, and X. Zhang, "Numerical and experimental investigation of active vibration control in a cylindrical shell partially covered by a laminated PVDF actuator," Smart Materials and Structures, vol. 17, no. 3, Article ID 035024, 2008.

[3] X. T. Cao, L. Shi, X. S. Zhang, and G. Jiang, "Active control of acoustic radiation from laminated cylindrical shells integrated with a piezoelectric layer," Smart Materials and Structures, vol. 22, no. 6, Article ID 065003, pp. 3-18, 2013.

[4] W. P. Li and H. Huang, "Integrated optimization of actuator placement and vibration control for piezoelectric adaptive trusses," Journal of Sound and Vibration, vol. 332, no. 1, pp. 17-32, 2013.

[5] Y. Zhu, J. Qiu, H. Du, and J. Tani, "Simultaneous optimal design of structural topology, actuator locations and control parameters for a plate structure," Computational Mechanics, vol. 29, no. 2, pp. 89-97, 2002.

[6] Z. Kang and L. Tong, "Integrated optimization of material layout and control voltage for piezoelectric laminated plates," Journal of Intelligent Material Systems and Structures, vol. 19, no. 8, pp. 889-904, 2008.

[7] B. Xu, J. S. Jiang, and J. P. Ou, "Integrated optimization of structural topology and control for piezoelectric smart trusses using genetic algorithm," Journal of Sound and Vibration, vol. 307, no. 3-5, pp. 393-427, 2007.

[8] B. Xu, J. P. Ou, and J. S. Jiang, "Integrated optimization of structural topology and control for piezoelectric smart plate based on genetic algorithm," Finite Elements in Analysis and Design, vol. 64, pp. 1-12, 2013.

[9] W.-S. Hwang and H. C. Park, "Finite element modeling of piezoelectric sensors and actuators," AIAA journal, vol. 31, no. 5, pp. 930-937, 1993.

[10] S. Y. Wang, K. Tai, and S. T. Quek, “Topology optimization of piezoelectric sensors/actuators for torsional vibration control of composite plates," Smart Materials and Structures, vol. 15, no. 2, pp. 253-269, 2006.

[11] O. Sigmund, "Morphology-based black and white filters for topology optimization," Structural and Multidisciplinary Optimization, vol. 33, no. 4-5, pp. 401-424, 2007. 

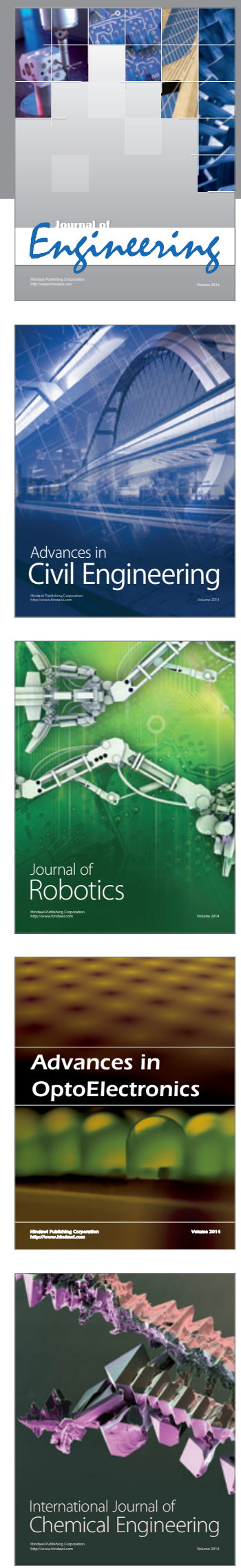

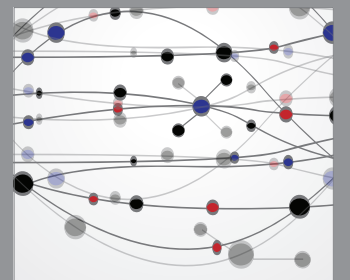

The Scientific World Journal
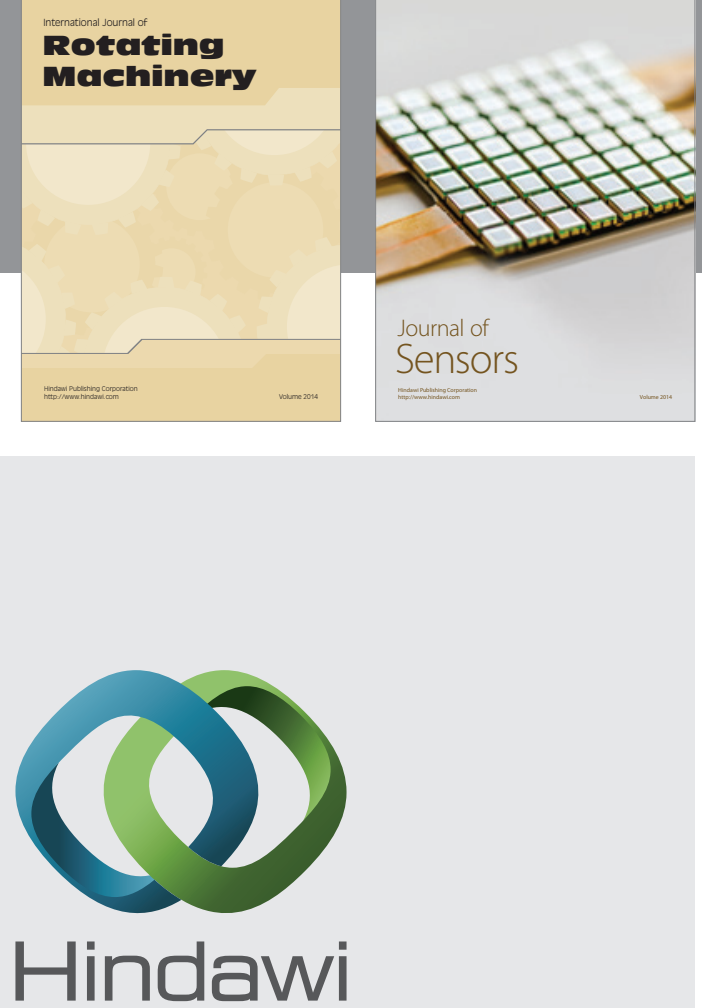

Submit your manuscripts at http://www.hindawi.com
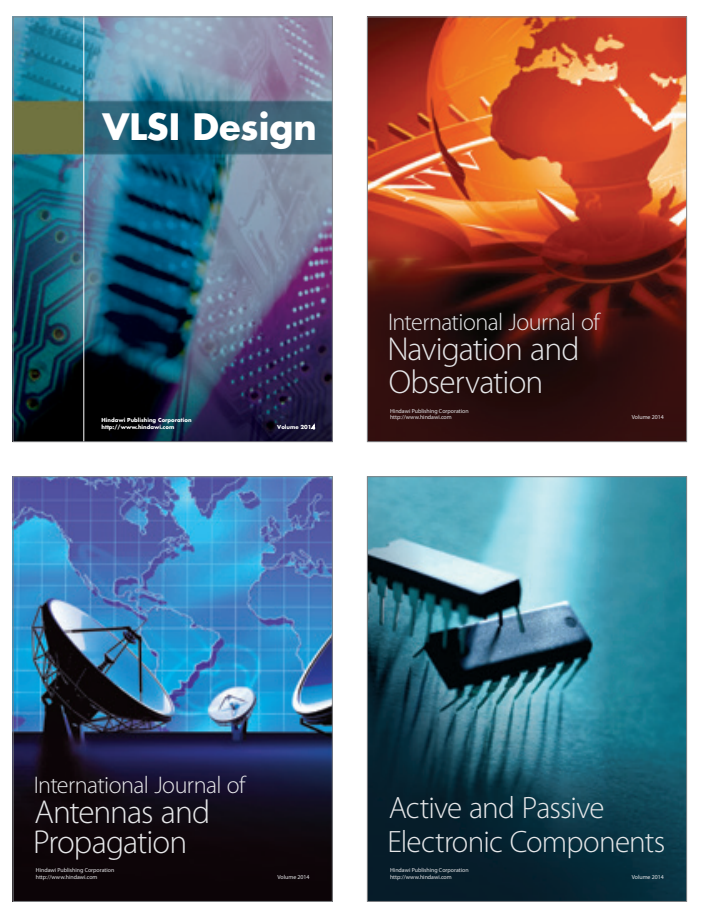
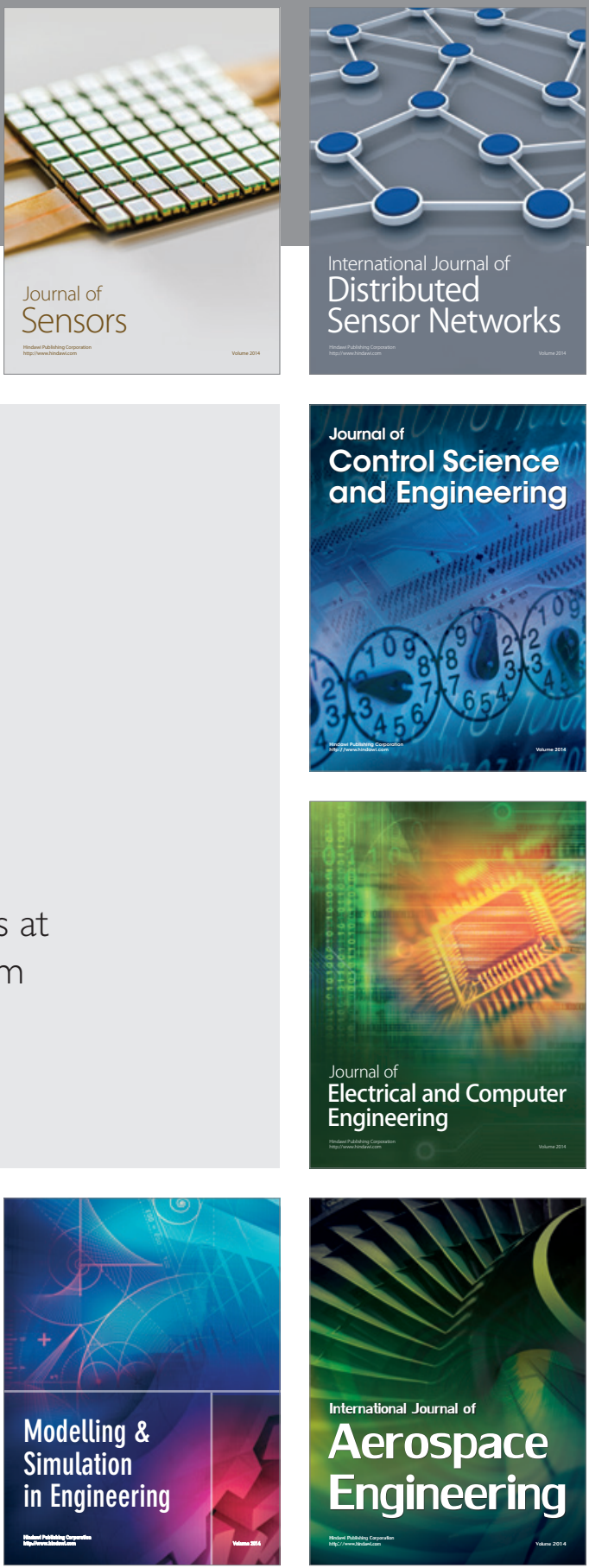

Journal of

Control Science

and Engineering
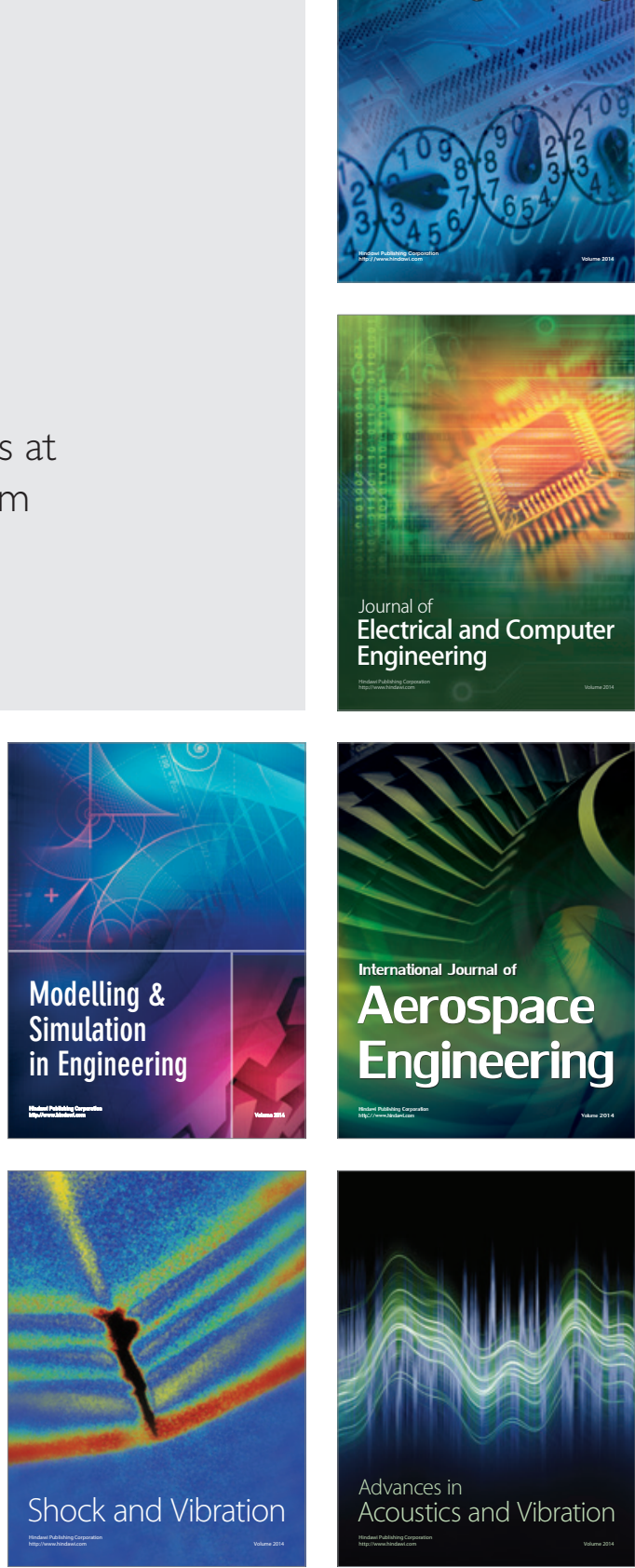